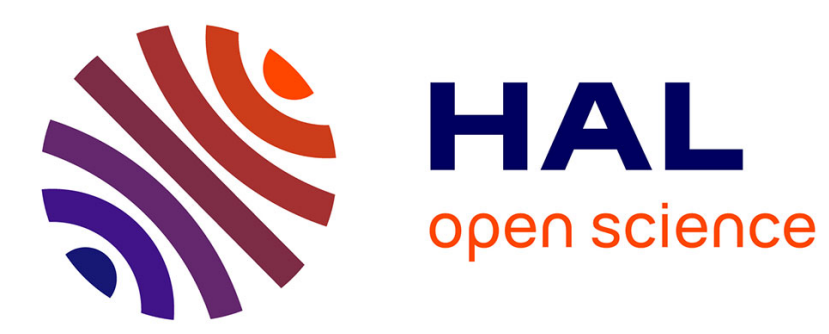

\title{
La construction mimétique du soi-agent et les phénomènes de schizophrénie débutante
}

\author{
L.-L. Salvador
}

\section{To cite this version:}

L.-L. Salvador. La construction mimétique du soi-agent et les phénomènes de schizophrénie débutante. Annales Médico-Psychologiques, Revue Psychiatrique, 2008, 166 (8), pp.620-626. 10.1016/j.amp.2005.09.030 . hal-03258513

\section{HAL Id: hal-03258513 \\ https://hal.science/hal-03258513}

Submitted on 11 Jun 2021

HAL is a multi-disciplinary open access archive for the deposit and dissemination of scientific research documents, whether they are published or not. The documents may come from teaching and research institutions in France or abroad, or from public or private research centers.
L'archive ouverte pluridisciplinaire HAL, est destinée au dépôt et à la diffusion de documents scientifiques de niveau recherche, publiés ou non, émanant des établissements d'enseignement et de recherche français ou étrangers, des laboratoires publics ou privés. 


\title{
La construction mimétique du soi-agent et les phénomènes de schizophrénie débutante
}

\section{The mimetic construction of the 'agent self' and the onset of schizophrenia}

\author{
Luc-Laurent Salvador \\ LIRDEF \\ Composante: Didactique et Socialisation - Équipe d'Accueil n`3749 \\ IUFM \& Université Montpellier II
}

\section{Résumé}

e-mail:salvador@ext.jussieu.fr

Une nouvelle interprétation des phénomènes de schizophrénie débutante est ici proposée sur la base d'un modèle mimétique et attributionnel du soi-agent qui permet de concevoir une construction du soi unitaire car sociale de bout en bout, y compris dans la dimension d'agence. Le vécu caractéristique du sujet qui en vient à se sentir au centre de l'attention de tous y apparaît comme la version pathologique d'une " centralité » normale du soi relative à ce qu'il est convenu d'appeler le "miroir social ». L'hypothèse présentée ici est qu'un déficit d'attributions causales de la part de l'entourage tend à susciter une abréaction du schème du soi qui est alors porté à "halluciner » des signes d'attributions dans les moindres attitudes, attentions ou messages de l'entourage. Signes venant alimenter le soi non pas sous le rapport de l'estime de soi mais sous le rapport du sentiment d'agence nécessaire pour se sentir exister comme être animé, comme être capable d'un agir sur le monde. Un des avantages de cette conception est qu'elle permet d'éclairer la fragilité des adolescents sous le rapport de la schizophrénie.

\footnotetext{
Abstract

A new interpretation of the onset of schizophrenia is presented which is based on a mimetic and attributional model of the self as agent. The typical feeling of being a focus of attention of an entourage sometimes extended to the whole world will appear as just the pathological version of the healthy "centrality" of the self in the "social mirror ». The hypothesis is that if the causal attributions that normally "feed" the self along the agency dimension are too scarce, it will lead the self schema to an "abreaction" which consists of "hallucinating" personal causal attributions in the minute attentions, attitudes and messages provided by anonymous public social interactions. All these signs, when perceived and delusionally interpreted, will help the subject maintain the vital feeling of being a "cause" capable of "effects" which characterizes animated beings as opposed to pushed and pulled pawns. This conception might shed some new light on the specific susceptibility of adolescents regarding schizophrenia.
}

Mots-clés : imitation, attributions, sentiment d'agence, miroir social, intersubjectivité

Key-words : imitation, attributions, feelings of agency, social mirror, intersubjectivity 


\section{Introduction}

Dans une importante série de travaux faisant suite à des recherches portant sur les biais attributionnels défensifs de sujets normaux [17,42], Bentall et ses collègues ont tenté d'étayer l'hypothèse selon laquelle les sujets présentant un délire de persécution ${ }^{1}$ seraient amenés à tenir leur entourage pour responsable de leur situation afin de préserver une estime de soi fragilisée.

Aussi judicieuse et nécessaire qu'ait été cette mise en évidence d'un lien particulier entre style attributionnel et fragilité de l'estime de soi chez les sujets en délire de persécution, il apparaît difficile d'y voir, comme Bentall [3], l'alpha et l'omega de la psychose. D'abord, parce qu'il est douteux que la saisie du soi puisse être réalisée sous le seul angle de l'estime de soi, construct très spécifique et néanmoins problématique tant du point de vue conceptuel que du point de vue de l'opérationnalisation $[2,4]$. Ensuite, parce que de nombreux éléments du tableau psychotique ne sont pas pris en compte par cette explication. On peut penser en particulier à la phénoménologie de la schizophrénie débutante dont la dimension subjective ou existentielle est très tôt apparue comme déterminante. Le sujet entrant en schizophrénie vit, en effet, un profond bouleversement de son rapport au monde et, particulièrement, de son rapport aux autres $[7,12,29,40]$. Or, Bentall n'en dit rien. Sa perspective solipsiste, conforme à ce qui a longtemps été un des travers majeurs du courant cognitiviste, réduit l'autre à un simple locus d'attributions externes, elle en fait la cible fantômatique d'une logique unidirectionnelle qui, étrangère à toute réciprocité, laisse échapper ce qui se joue de sujet à sujets. Pourtant, de quelque manière qu'on la conçoive, l'intersubjectivité est une dimension qui, bien que s'étant longtemps trouvée dans la tâche aveugle du behaviorisme puis du

\footnotetext{
1 Relevant aussi bien de la schizophrénie paranoïde que du délire paranoïaque.
} 
cognitivisme, ne peut plus être actuellement négligée, et cela, que l'on concède ou non l'existence de ce qui pourrait s'énoncer comme cette « imitation générale » que nous évoquerons plus bas.

C'est précisément parce qu'il se situait, au moins à l'origine, dans le contexte de l'anthropologie girardienne que Grivois [12-14] en est venu à interpréter la schizophrénie débutante comme résultant d'un dérèglement $d u$ mimétisme.

Dans ce qui suit, je m'efforcerai de montrer que son hypothèse, bien que mettant en avant avec raison l'existence d'une thématique mimétique jusqu'alors négligée, ne le fait pas au niveau pertinent car en affirmant qu'il s'agit avant tout d'un dérèglement moteur, Grivois en vient à révoquer explicitement la dimension sociale — et, implicitement, la dimension psychologique. Or, il conviendrait aussi de repérer cette dynamique mimétique au niveau psychologique le plus sophistiqué, celui de la cognition (réellement) sociale dont relèvent ces attributions causales auxquelles Bentall s'est attaché. Car c'est précisément au point de confluence de ces deux logiques, mimétique et attributionnelle qu'il est possible de saisir le soi dans son unité, c'est-à-dire, tout à la fois sous l'angle du soi-objet (le moi) et celui du soi-agent (le je) $[34,36]$.

L'hypothèse proposée est qu'en effet le soi serait construit par la reproduction, l'intériorisation ou, plus simplement, l'imitation des attributions causales et acausales inhérentes au «miroir social» et qu'en tant que tel, il susciterait la phénoménologie d'entrée en schizophrénie lorsque placé en état de «sous-alimentation» chronique en attributions. Il se mettrait alors, en quelque sorte, à « halluciner » des marques d'attention et donc d'attributions le 
concernant de la part d'un entourage réel ou imaginaire. Ceci permettrait de réinstaurer le lien social absent chez Bentall en replaçant son attributeur solipsiste face à une foule, la foule des autres, perçus comme unanimes et qui, bien qu'aussi porteurs de ces modèles comportementaux qui ont intéressé Grivois, sont avant tout pourvoyeurs d'attributions susceptibles d'être assimilées par la dynamique mimétique constructrice du soi.

Cette conception centrée sur un soi construit socialement, mimétiquement, notamment dans sa dimension d'agent, est purement psychologique, mais elle n'exclut pas, bien évidemment, l'intervention de niveaux de causalité sousjacents étant donné qu'ils sont susceptibles d'affecter les fonctions psychologiques évoquées ici.

\section{L'entrée en schizophrénie}

Les phénomènes de schizophrénie débutante présentent un aspect caractéristique lié au sentiment qu'a le sujet d'être concerné par les attitudes, comportements et discours des autres $[7,12,29,40]$. Ce temps du concernement [12-14] avait été baptisé trema par Conrad [7], terme que l'on peut traduire par « trac » et qui renvoit, précisément, à cette situation bien connue où, sans être avec les autres, un sujet perçoit les signes de leur présence et se les représente, avec autant d'angoisse que d'exhaltation, comme n'ayant d'autre intention que celle de porter attention sur lui pour l'évaluer, le juger : « le monde regarde le patient avec une physionomie qui le met en question » (Conrad, trad. [37]).

Par «monde », il faut ici, bien sûr, entendre les autres, tous les autres, ce qui veut dire qu'on se situe déjà à la limite supérieure du concernement, lorsque celui-ci est généralisé et que la mise en question du sujet devient radicale. Situation qui ouvre sur ce que Grivois désigne comme la centralité, 
atteinte lorsque, face à ce constat d'unanimité, le sujet en vient à la conviction qu'il a un rôle à jouer, sans nécessairement savoir lequel. Ce qui correspond chez Conrad à la phase dite apophanique au cours de laquelle la signification de ce qui est vécu s'installe, ou plutôt, se révèle. ${ }^{2}$

On a donc là deux phases articulées de manière logique puisque la première correspond en quelque sorte à une accumulation de données qui posent question au sujet et que la seconde correspond à une interprétation visant à faire sens de cet ensemble de données et constitutive du délire.

Selon Grivois, ce que le sujet repère avant tout, ce sont des similitudes comportementales qui se présenteraient de manière incessante et dont la généralité exclurait toute possibilité de hasard. Face à cet emballement mimétique, le sujet en conclut très vite qu'il est au centre de l'attention des autres. A l'évidence, « ils » font cela « de concert». Mais pour quelle raison le font-ils ? Telle est la question qu'il se pose et à laquelle il tentera de répondre par des interprétations que l'on peut considérer comme constitutives du délire.

Grivois situera l'origine de ce concernement dans la «surchauffe » d'un mimétisme moteur fondamental qui, selon lui, se trouverait à l'œuvre de manière tout à fait normale et générale dans les processus de coordination sociale les plus élémentaires, c'est-à-dire, ceux qui, jouant au niveau corporel, opèrent lorsque, par exemple, un certain nombre de personnes se croisent anonymement dans un même espace. Cette «imitation de pure motilité » n'aurait d'autres aliments que les éléments posturaux ou gestuels saisis chez les autres sans y prêter attention et donc, sans intention, sans conscience autre que fugitive.

\footnotetext{
2 Dans le moment dit de l'anastrôphé, terme dont l'étymologie renverrait à la centralité [39]
} 
Toutefois, dans ce contexte d'un mimétisme moteur supposé non conscient, général et normal, il est difficile d'imaginer comment une quelconque surchauffe mimétique pourrait apparaître et venir perturber le champ phénoménologique du sujet. Grivois reconnaîtra cette difficulté, qui vient de ce que davantage de conformité ne fait pas sortir de la conformité, mais il restera sans solution satisfaisante. Focalisé sur l'aspect moteur au point d'exclure toute dimension sociale du processus de schizophrénie débutante, Grivois délaisse en fait tout un ensemble de données ne relevant pas de la seule dimension motrice comme ces similitudes repérées au niveau de l'apparence physique ou ces messages occupant l'espace public (publicités affichées, émissions radiophoniques et télévisuelles) auxquels ses patients viennent pourtant alimenter leur sentiment d'être au centre de l'attention de tous. Or, nous allons le voir, le registre mimétique ne se limite pas, loin s'en faut, au seul plan de la motricité.

\section{L'hypothèse mimétique}

L'imitation connaît, en effet, depuis une vingtaine d'année un lent mais sûr regain d'intérêt dans un grand nombre de domaines allant de l'anthropologie à la robotique en passant par les neurosciences, l'économie et les différentes psychologies (pour une vision d'ensemble cf. [19]),

La découverte des neurones miroirs y a dernièrement beaucoup contribué. Ces neurones, actifs aussi bien quand un sujet exécute une action que lorsqu'il la voit accomplie par un congénère [33] constituent la trace biologique d'un lien soi-autre. Ils redonnent à la vieille notion de "résonance » une actualité inattendue et tire le mimétique vers les niveaux les plus fondamentaux de la psyché humaine. 
Nous nous situerons dans ce contexte où l'imitation apparaît comme centrale dans la genèse du fait humain $[1,9,11,25,41]$. Ainsi que cela a été montré par ailleurs [34,35] les conceptions cycliques de l'organisation psychologique $[1,32,44,45]$ permettent de voir celle-ci comme une population de schèmes ou d'habitudes qui, tendant à se reproduire, apparaissent comme autant d'oscillateurs dont la résonance instancie une mécanique de l'imitation — ces deux derniers termes étant chacun à entendre en un sens générique et non spécifique. Sauf à être inhibée ou réprimée, cette tendance nous porte à reproduire tout ou partie de l'activité mentale ou comportementale d'un modèle suite à la perception ou la représentation de ladite activité. Perspective qui, bien sûr, englobe les processus dits de l'«intersubjectivité », comme l'empathie, la sympathie et toutes les formes de « role-taking », dans lesquelles un sujet se met «à la place de l'autre», opérant par ce fait même une reproduction constitutive du fait intersubjectif, mais aussi, nous allons le voir, constitutive du fait subjectif lui-même.

\section{La construction mimétique du soi-agent}

Cette tendance mécanique et donc générale à l'imitation va bien au-delà de la seule motilité. On la retrouve en particulier au fondement de ce qui, supposément, en constitue la négation la plus radicale : le soi, dont nous allons tenter à présent d'analyser le rôle dans les processus schizophréniques à partir d'un modèle mimétique et attributionnel du soi-agent [34,36] dérivé de la notion bien connue de « miroir social ».

Née bien avant que James [20] introduise la distinction classique entre, d'une part, le soi-objet, le moi, et le soi-agent, le je, cette idée selon laquelle un sujet en viendrait à se connaître et, plus exactement, à s'évaluer, en se 
mirant dans le regard que les autres portent sur lui est à l'origine d'un large courant de recherche. C'est le sociologue Cooley qui en a donné la formule canonique avec l'hypothèse dite du soi-miroir (looking-glass self): «Lorsqu'en présence d'une personne pour laquelle nous sentons que nous avons de l'importance, nous avons tendance à nous figurer et à adopter par sympathie le jugement qu'elle porte à notre égard. » [8].

Reprise par l'interactionnisme symbolique [30], testée en laboratoire comme en situation naturelle, cette idée que les sujets tendent à conformer la perception qu'ils ont d'eux-mêmes aux feed-backs fournis par leur entourage social a été amplement validée [15,16,38].

Elle est toutefois restée cantonnée au soi-objet, c'est-à-dire, au soi en tant qu'objet de connaissance et/ou objet d'une évaluation, l'estime de soi en étant, sans doute, le construct le plus représentatif. C'est pourquoi, il a semblé judicieux d'étendre cette perspective au soi dans sa dimension d'agent, et surtout, de dépasser le stade de la simple métaphore, en considérant le soimiroir comme consistant (a) à se re-présenter les attributions (jugement) des autres à son égard puis (b) à entériner, à faire sienne, donc à imiter, cette attribution. En prenant en compte la dimension causale des attributions, cette relecture attributionnelle ouvrait la possibilité d'une genèse sociale de cet insaisissable soi-agent, le je, conçu alors comme résultant de l'intériorisation ou de l'imitation des attributions causales opérées, souvent implicitement, par l'entourage à l'égard du sujet [34,36]. Par exemple, dire de Pierre qu'il est coléreux parce qu'il vient de casser un vase intentionnellement, c'est aussi reconnaître tacitement sa responsabilité dans la destruction du vase. L'attribution au soi-objet du trait de personnalité «coléreux » est donc 
porteuse d'une attribution causale implicite qui confère un statut d'agent à Pierre. Statut qu'il pourra directement intérioriser, imiter, pour se penser comme tel, agent, c'est-à-dire, source causale d'un effet sur le monde.

Depuis les routines mère-enfant jusqu'aux interactions professionnelles en passant par tout le processus éducatif, le «miroir social» nous assigne ainsi constamment un statut de cause vis-à-vis des effets de notre action dans le monde et ce, indépendamment de leur valeur positive ou négative.

Cette perspective suggère que des relations sociales appauvries entraînant, de ce fait, une raréfaction de ces précieuses attributions - puisse placer le schème du soi-agent en situation de déprivation. L'expression piagétienne « les schèmes ont faim » devient ici lourde de sens et nous amène à postuler la possibilité d'un abréaction du schème du soi.

\section{L'hypothèse d'abréaction du schème du soi}

Cette notion renvoit ici aux travaux de Lorenz [27] qui cherchant à démontrer l'existence de comportements innés chez l'animal, plaça en situation d'environnement drastiquement appauvri divers sujets animaux qui, malgré tout, manifestèrent lesdits comportements en « hallucinant» les matériaux manquants sur leur propre corps. Les coqs, canards ou poissons se mettaient à agresser leur propre queue comme s'il s'était agi d'un congénère. Les rats se mettaient à construire un nid en utilisant le bout de leur queue. Les écureuils ou les chiots effectuaient des enfouissements « dans le vide ». Selon Lorenz, tous les comportements seraient portés à s'exécuter en fonction d'une motivation qui croîtrait au fur et à mesure du temps écoulé depuis la dernière exécution, un peu comme si de l'eau s'accumulait dans un réservoir et en augmentait progressivement la pression jusqu'à amener l'ouverture de la vanne 
— l'exécution du comportement — même en l'absence des stimuli environnementaux habituels.

Ce modèle hydraulique, parce qu'il est conforme au schématisme piagétien, suggère la possibilité qu'un «schème du soi » insuffisamment alimenté en attributions causales puisse se réguler en abaissant ses seuils et en récupérant les moindres éléments attributionnels disponibles, un peu comme «faute de grives, on mange des merles ».

De fait, il semblerait que les sujets versant dans la schizophrénie soient très attentifs aux interactions «minuscules » se jouant de manière anonyme et habituellement insignifiante dans une sphère publique qui en regorge. Par exemple, les coordinations motrices élémentaires évoquées par Grivois peuvent être lues comme autant de signaux indiquant à l'autre qu'il est reconnu comme être animé et non pas confondu avec les choses inertes, immeubles et mobiliers peuplant l'espace public. Cette simple attention à l'autre véhicule une attribution causale $[10,43]$ que nous percevons mais qui n'émerge à la conscience que lorsqu'elle fait défaut, lorsque, par exemple, nous nous trouvons bousculés par un rustre qui ne daigne même pas s'excuser. Nous nous sentons alors, aussi peu que ce soit, blessés, d'avoir été traité ainsi, comme si nous n'étions pas une personne, mais un objet malencontreusement heurté.

L'espace public est aussi envahi par des médias ne cessant de nous adresser des messages, publicitaires ou autres, qui, en tant qu'ils manifestent une communication et donc, un échange de personne à personne, véhiculent une forme de reconnaissance de leur audience à laquelle nous ne prêtons plus attention mais qui, bien réelle, pourra être assimilée par un schème du soi « affamé ». 
Il faut bien voir qu'étant habituellement ignoré, ce "bruit de fond» attributionnel, une fois découvert, va nécessairement bouleverser le champ phénoménologique du sujet concerné qui, dans une démarche des plus rationnelles, va activement rechercher une explication. Mais le bon sens est ici pris au dépourvu. Ces micro-attributions, par leur multiplicité, leur universalité apparente et donc, leur unanimité de fait, poussent le sujet à une hypothèse folle, incroyable : c'est sur lui que les membres de la foule se coordonnent, il est concerné, c'est lui qui est en cause. Sentiments mégalomanes et exhaltations missionaires — sentiments d'agence exarcerbés — naissent ici, dans ce jeu de miroir impensable pour notre culture individualiste qui, obstinément, scotomise le mimétique.

De la centralité normale du «miroir social » à la centralité pathologique, le processus reste invariant. Seul varie le paramètre constitué par l'intensité du besoin d'assimiler des attributions, le besoin d'être objet d'attention, qui dépend de la qualité du «miroir social » dont le sujet bénéficie. Lorsque ce miroir se dégrade, s'assombrit ou disparaît, le sujet se retrouve en état de manque plus ou moins durable pouvant aller jusqu'à la carence. Il se trouve alors fragilisé, et susceptible d'entrer en abréaction du schème du soi. Nous verrons plus loin en quoi cela correspond en particulier au cas de l'adolescent, mais nous pourrions évoquer aussi celui de l'expatrié, voyageur ou migrant.

\section{La thèse attributionnelle de Bentall}

Dans une importante série de recherches, Bentall et ses collègues [3-5,2123] ont défendu une hypothèse qui pourrait sembler venir directement à l'appui de la présente conception puisqu'elle porte sur l'existence de stratégies attributionnelles de protection de l'estime de soi chez les sujets délirants. Ces 
travaux confortent en effet l'idée que, d'une part, la fragilité du soi est en cause, et que, d'autre part, une dynamique attributionnelle est à l'œuvre. Cependant, dès qu'on y regarde de plus près, le tableau est beaucoup moins simple qu'il n'y paraît car le soi n'y est appréhendé qu'au travers du construct « estime de soi » dont le niveau, chez les sujets atteints d'un délire de persécution, était très variable d'une étude à l'autre.

Tentant de tirer cela au clair, Bentall et ses collègues ont pu démontrer l'existence chez ces sujets d'une faible estime de soi latente associée à une estime de soi normale ou élevée lorsqu'évaluée sur des questionnaires conventionnels et donc explicites [22,26,28]. Conclusion qui corrobore leur hypothèse d'une stratégie défensive de l'estime de soi. Mais par ailleurs, s'appuyant sur la théorie de la «self-discrepancy» [18], ces chercheurs ont aussi fait apparaître que les sujets présentant un délire de persécution affichent une bonne congruence entre leur soi actuel et leur soi idéal, en même temps qu'un fort décalage entre le soi actuel et l'image d'eux-mêmes que, selon eux, leurs parents entretiennent [22].

Etrangement, ce dernier résultat, qui révèle l'existence d'une mauvaise image — explicite et non latente — du soi « perçu dans le regard des autres », n'a pas été pas reconnu par Bentall comme manifestant une faible estime du soi social. Ce dont, supposément, le délirant tenterait de se protéger mais qui pourtant découle nécessairement d'un style attributionnel tendant à incriminer un entourage jugé malveillant! On voit ainsi que l'hypothèse de Bentall se trouve en difficulté précisément au point où elle intercepte le miroir social, le regard des autres. 
Ceci nous amène à envisager l'existence d'une autre motivation que la simple sauvegarde de l'estime de soi. Une motivation plus fondamentale encore puisqu'opérant aux dépens de cette dernière. Il se pourrait en effet que le délire de persécution n'ait pas tant pour visée de préserver l'estime du soi — qui relève du soi-objet — que de sauver le soi d'une déstructuration bien plus grave, car relative au sentiment d'agence. Le fait que le sujet en phase de trema ou de concernement s'installe dans une représentation de son entour social comme lui portant une attention généralement malveillante pourrait venir de ce que cette attention véhicule un aliment attributionnel lui permettant de se sentir être de la manière la plus essentielle, en tant qu'agent et ce, au-delà de toute espérance, d'où les délires missionnaires. Il se pourrait donc que ce ne soit pas tant le soi-objet et le construct d'estime de soi servant à l'appréhender qui soient en question dans le délire de persécution, mais plutôt le soi-agent, plus essentiel, plus fondamental puisque fondateur de l'être en tant qu'il serait animé et non pas chose. Enjeu bien plus vital qui explique que, contrairement à l'hypothèse de Bentall, l'image de soi puisse lui être sacrifiée.

Ce type de réaction ne nous est pas aussi étrangère qu'il y paraît. Elle est en effet comparable au cas bien connu de l'élève turbulent qui persévère dans un comportement ayant pour seules conséquences visibles de lui faire subir des reproches et des sanctions normalement aversifs. Comme chacun, au fond, le sait bien, le bénéfice de cet élève, dont il a la jouissance immédiate et dont on peut penser qu'il en devient dépendant, c'est ce sentiment jubilatoire d'exister qui se nourrit de l'attention que tous lui portent unanimement, enseignant compris. Car cette attention, même accompagnée de la plus totale réprobation, véhicule des attributions causales qui alimentent le soi-agent en le 
reconnaissant capable d'un effet sur le monde, capable de nuisances à défaut d'excellence.

\section{Discussion}

Par la mise en avant du soi-agent, l'hypothèse proposée ici opère en quelque sorte une coordination entre la perspective mimétique de Grivois et celle, attributionnelle de Bentall et souligne en même temps leur commune incomplétude sous le rapport du soi. Incomplétude qui explique probablement que ces deux thèses n'aient rien eu à dire de la particularité de l'adolescence sous le rapport de la schizophrénie. Alors que l'hypothèse d'un soi-agent mimétiquement construit permet d'en faire sens de manière assez immédiate.

En effet, bien que difficile à circonscrire avec précision, l'adolescence se définit a minima comme un passage entre l'enfance et l'état adulte, toute la question étant de savoir comment exprimer au plus juste cette idée. Dans les termes de la psychologie mimétique du soi précédemment exposée, elle signifie, pour l'essentiel, que l'adolescent va devoir opérer une transition entre, d'une part, un « miroir social » constitué avant tout par le cercle familial — qui fut pourvoyeur premier et plus ou moins satisfaisant d'attributions, mais qui ne peut le rester indéfiniment et perd donc progressivement son pouvoir nourricier - et d'autre part, un «miroir social» à construire dans l'environnement social élargi qui deviendra, à terme, le principal pourvoyeur d'«aliments attributionnels». Quel que soit le régime attributionnel ayant émergé de l'histoire des interactions du système enfant-famille, cette transition fragilisera l'adolescent. Autrement dit, tous les adolescents sont concernés à des degrés divers par cette fragilisation. Mais, bien entendu, celle-ci mettra surtout en danger les plus faibles qui, n'ayant pu ou su construire un régime 
d'attributions d'origine familiale satisfaisant, manquant d'allant, de confiance et de compétences sociales, pourront plus facilement glisser vers le retrait et l'isolation au point de se retrouver, selon une expression de Bentall, « coincés dans le no man's land entre l'enfance et le monde adulte ».

On comprend alors que les traits caractéristiques de l'adolescence — qui dessinent un sujet en quête tout à la fois de modèles et d'identité, chaviré par d'importantes oscillations, tant émotionnelles que d'estime de soi, et qui en vient à se centrer de manière angoissée sur lui-même via le regard des autres puissent être évocateurs des prodromes comme du processus d'entrée en schizophrénie. Les deux premières phases de la schizophrénie débutante sont, de fait, aisément perceptibles dans la définition de l'égocentrisme adolescent de Lapsley \& Quintana : «L'égocentrisme adolescent se manifeste au travers de deux élaborations, l'audience imaginaire et la légende personnelle. L'audience imaginaire, c'est le fait que l'adolescent croit que les autres sont aussi préoccupés par ses comportements et son apparence qu'il l'est lui-même. La deuxième composante, la légende personnelle, est porteuse du sentiment d'unicité et d'indestructibilité de sa personne. » [24].

\section{Conclusion}

Il a été proposé ici un modèle de la schizophrénie comme résultant d'un schème du soi carencé en attributions causales de la part du miroir social et entrant en abréaction, c'est-à-dire, portant attention à ces attributions causales « minuscules » et habituellement non perçues qui occupent la sphère publique. Ce modèle, centré comme il l'est sur la question du soi saisi dans sa dimension d'agent, s'accorde pleinement avec la position de l'approche phénoménologique selon laquelle la schizophrénie serait «une affection 
bouleversant la personne humaine tout entière, sa façon «d'être au monde » [6]. Le soi-agent, le je, représente en effet la part quintessentielle de soi, celle que nous ne pouvons jamais proprement tenir, contrairement au soi-objet, mais à laquelle nous ne renonçons jamais — sauf à connaître une extraordinaire expérience initiatique et/ou mystique - et au travers de laquelle nous pensons notre place et notre action dans le monde.

A l'écart de l'effervescence paradigmatique qui pousse à faire feu de tout construct cognitiviste, mais trop souvent au ras des données empiriques, il a semblé préférable de procéder à quelques "pas en arrière » pour tenter de rapprocher la psychologie, et notamment la psychopathologie, du champ plus vaste de l'anthropologie, notamment girardienne. A ma connaissance, celle-ci est la première à avoir articulé ces dynamiques mimétique et attributionnelle que l'on trouve au cœur du processus de construction du sujet.

L'utilité de ce rapprochement pourrait s'affirmer non seulement au plan conceptuel mais aussi au plan thérapeutique au travers d'interventions psychoéducatives qui, en éclairant les sujets sur les dimensions mimétique et attributionnelle des réalités humaines en général, du soi en particulier, pourraient probablement les aider à recadrer ou à réélaborer ces perceptions inquiétantes qui se présentent au seuil de la schizophrénie et vont ensuite alimenter le délire. Seules des investigations pourront décider du bien-fondé de cette proposition, mais l'impact des psychoéducations étant déjà assez bien avéré [31], on peut avoir quelques raisons d'espérer. Avec prudence toutefois, car l'éducation au fait mimétique allant frontalement à l'encontre du zeitgeist individualiste, elle pourrait susciter un certain nombre d'obstacles, de réactances, de la part de sujets peut-être peu enclins à faire le deuil d'un certain 
nombre d'illusions proprement romantiques. Ainsi que Nietzsche l'avait si lucidement affirmé dans Humain, trop humain : « La connaissance la plus forte (celle de l'absolue non-liberté de la volonté humaine) est pourtant celle qui aboutit aux résultats les plus pauvres : car elle a toujours eu l'adversaire le plus fort, la vanité humaine. »

\section{9. références}

[1] Baldwin JM. Mental development. New York : MacMillan ; 1895.

[2] Baumeister RF, Smart L, Boden JM. Relation of threatened egotism to violence and aggression: The dark side of high self-esteem. Psychological Review 1996 ; 103 : 5-33.

[3] Bentall RP. Madness explained: Psychosis and human nature in medicine. London : Allen Lane ; 2003.

[4] Bentall RP, Corcoran R, Howard R, Blackwood N, Kinderman P. Persecutory delusions: a review and theoretical integration. Clinical Psychology Review 2001 ; 21 : 1143-1192.

[5] Bentall RP, Kinderman P., Kaney S. The self, attributional processes and abnormal beliefs: Towards a model of persecutory delusions. Behaviour Research \& Therapy 1994 ; 32 : 331-341.

[6] Bovet P, Gamma F. Peut-on définir la notion de vulnérabilité à la schizophrénie sous un angle psychodynamique ? In Petitjean F, Bokobza H, Botbol M, Caroli F, Glikman J, Marie-Cardine M, et al., Eds. Schizophrénies débutantes. Montrouge : John Libbey Eurotext, 2003. p. 79-87.

[7] Conrad K. Die beginnende schizophrenie. Versuch einer gestaltanalyse des Wahns. Stuttgart: Thieme ; 1958.

[8] Cooley $\mathrm{CH}$. Human nature and the social order. New York : Transaction Publishers ; 1902.

[9] Donald M. Les origines de l'esprit moderne : trois étapes dans l'évolution de la culture et de la cognition. Bruxelles : De Boeck; 1999.

[10] Duval S, Wicklund RA. A theory of objective self awareness. New York : Academic Press ; 1972.

[11] Girard R, Oughourlian JM, Lefort G. Des choses cachées depuis la fondation du monde. Paris : Grasset ; 1978.

[12] Grivois H. Naître à la folie. Paris : Empêcheurs de penser en rond ; 1991.

[13] Grivois H. Urgence folie. Paris : Empêcheurs de penser en rond ; 1993.

[14] Grivois H. Le fou et le mouvement du monde. Paris : Grasset ; 1995.

[15] Harter S. The construction and conservation of the self: James and Cooley revisited. In : Lapsley DK, Power FC, Eds. Self, ego and identity. New York : Springer Verlag, 1988 p. 43-70.

[16] Harter S. Visions of self: Beyond the Me in the Mirror. Nebraska Symposium on Motivation $1993 ; 40: 99-144$. 
[17] Heilbrun A, Bronson N. Fabrication of delusional thinking in normals. Journal of Abnormal Psychology $1975 ; 84$ : 422-425.

[18] Higgins ET. Self-discrepancy: A theory relating self and affect. Psychological Review 1987 ; 94 : 319-340.

[19] Hurley S, Chater N. Eds. Perspectives on Imitation: From Neuroscience to Social Science. Cambridge, MA: MIT Press ; 2005.

[20] James W. The principles of psychology. New York : Holt ; 1890.

[21] Kaney S, Bentall RP. Persecutory declusions and attributional style. British Journal of Medical Psychology 1989 ; 62 : 191-198.

[22] Kinderman P, Bentall RP. Self-discrepancies and persecutory delusions: Evidence for a defensive model of paranoid ideation. Journal of Abnormal Psychology 1996 ; 105 : 106-114.

[23] Kinderman P. Attentional bias, persecutory delusions and the self concept. British Journal of Medical Psychology 1994 ; 67 : 53-66.

[24] Lapsley DK, Quintana SM. Integratives themes in social and developmental theories of self. In : Pryor TB, Day JD, Eds. The development of social cognition. New York : Springer Verlag, 1985 p. 152-176.

[25] Le Bon G. Psychologie des foules. Paris : Alcan ; 1895.

[26] Lee HJ. Attentional bias, memory bias and the self-concept in paranoia. Psychological Science $2000 ; 9$ : 77-99.

[27] Lorenz K. Evolution et modification du comportement. Paris : Payot ; 1965.

[28] Lyon HM, Kaney S, Bentall RP. The defensive function of persecutory delusions: Evidence from attribution tasks. British Journal of Psychiatry $1994 ; 164$ : 637-646.

[29] Mayer-Gross W. Die Klinik. In : Bumke O, Ed. Handbuch der Geisteskrankheiten, vol. 9: Die Schizophrenie. Springer, 1932 p. 293578.

[30] Mead GH. Mind, self, and society. Chicago : University of Chicago Press ; 1934.

[31] Petitjean F, Bokobza H, Botbol M, Caroli F, Glikman J, Marie-Cardine M, et al., Eds. Schizophrénies débutantes. Montrouge : John Libbey Eurotext; 2003.

[32] Piaget J. La Naissance de l'Intelligence chez l'Enfant. Neuchâtel : Delachaux \& Niestlé ; 1936.

[33] Rizzolatti G. The mirror neuron system and imitation. In : Hurley S, Chater N, Eds. Perspectives on Imitation: From Neuroscience to Social Science. Cambridge, MA : MIT Press, 2005. p. 55-76.

[34] Salvador LL. Imitation et Attribution de causalité : la construction mimétique du soi, la construction mimétique de la réalité. Applications à la psychose naissante et à l'autisme. Université Paris V : Thèse de doctorat non publiée ; 1996.

[35] Salvador LL. Assimilation, imitation and the elementary social fact. Toward a definition of social interactions. In : Working notes of the Socially Intelligent Agents Workshop, American Association for Artificial Intelligence (AAAI) Fall Symposium Serie. Redwood City : AAAI Press, 1997. p 115-117.

[36] Salvador LL. Imitation et attribution de causalité : la genèse sociale du soi-agent (en italien). In F. Braga Illa e G. Padovani (a cura) Processi di 
rappresentazione e teorie della mente. QuattroVenti : Urbino, 2001. p. 263-283.

[37] Seywert F, Célis-Gennart M. La transformation du champ de l'expérience dans la schizophrénie : l' « analyse structurelle » de Klaus Conrad. Evolution Psychiatrique 1999 ; 64 : 101-11.

[38] Shrauger JS, Schoeneman TJ. Symbolic interactionist view of selfconcept: through the looking glass darkly. Psychological Bulletin 1979 ; $86: 549-573$.

[39] Sirère S, Naudin J, Grivois H. La centralité comme mode de présence spécifique de l'expérience psychotique inaugurale. Annales MédicoPsychologiques 1999 ; 157 : 636-639.

[40] Sullivan HS. The onset of schizophrenia. American Journal of Psychiatry $1927 ; 6: 105-134$.

[41] Tarde G. Les lois de l'imitation. Paris : Alcan ; 1890.

[42] Taylor SE. Positive illusions. Creative self-deception and the healthy mind. New York : Basicbooks ; 1989.

[43] Taylor SE, Fiske ST. Salience, attention, and attribution : top of the head phenomena. Advances in Experimental Social Psychology 1978 ; $11: 249-288$.

[44] Uexküll J von. Mondes animaux et monde humain. Paris : Denoël ; 1934.

[45] Varela FJ. Autonomie et connaissance. Paris : Seuil ; 1989. 